As a reference book covering the classical era of investigations on these two vitamins, this volume cannot be faulted; experts in the field present authoritatively and clearly the basic information available up to 1964. It is only rarely that a reference for 1965 is encountered, so it appears that there has been a rather greater delay than usual these days between the completion of the manuscript and the publication of the volume. In spite of all the carefully compiled information, one is struck most forcibly by the enormous gaps that, with one outstanding exception-vision-still exist in our knowledge of the mode of action of vitamin A at the biochemical level. The same comment applies to ascorbic acid. There is here a great challenge to young biochemists, and the problems are clearly spelled out in chapters on "Biochemical Systems (Vitamin A)" by O. A. Roels, "Effects of Vitamin A Deficienoy in Animals" by T. Moore, and "Effects of Ascorbic Acid Deficiency in Animals" by G. C. Chatterjee. In an edited volume some variation in standard of coverage is inevitable, but in this case the editors have clearly done an excellent job in keeping authors as close to their brief as is humanly possible when dealing with scientists. Overlap is well controlled although the same diagram appears on pages 242 and 250.

If vitamin A-active carotenoids are to be considered together with vitamin A in later editions, they should be given fuller coverage than in the present volume; in this case it is so scant and out of date as to be occasionally misleading. In the ascorbic acid section some of the more modern aspects are not discussed in sufficient detail. For example, one is tantalized by the statement that "thin layer chromatography is also being tried out in ascorbic acid studies". One wonders with what success, especially as three references are added to this statement. These are minor points and this book will take its place as a worthy first volume of the new edition of The Vita. mins.

Volumes 6 and 7 began life 15 years ago as the well known and well tried Vitamin Methods. They now become the final volumes in the second edition of The Vitamins. This is a "take-over" which is logically justified. Volume 6 begins with an introductory chapter on "Animal Assays" by C. I. Bliss and P. Gyorgy and an excellent and detailed chapter on "Statistical Methods in Biological Assay of the Vitamins" by C. I. Bliss and C. White, and then continues to deal with each fat-soluble vitamin in detail. All these sections are thorough, critical, well informed and well documented. The chapter on vitamin $\mathbf{A}$ is perhaps not quite so up to date as the other chapters, but all the major assay procedures are fully described. Only methods for $\beta$-carotene are described in detail and little information is given on the separation and assay of other vitamin A-active carotenoids. It is significant that the chapter on vitamin $E$ by $R$. $H$. Bunnell is sufficiently up to date to describe the application of GLC techniques to assays of this vitamin and its congeners.

Volume 7 has been designed on a similar pattern to volume 6. It begins with an excellent, concise introductory chapter on "Principles of Microbiological Assay" by W. N. Pearson, and this is followed by expert discussions of all aspects of assaying water-soluble vitamins. A final chapter is concerned with "Clinical Evaluation of Malnutrition" by W. A. Krehl.

When one considers the three volumes together, one is lost in admiration for the hard work which the editors and contributors have put into compiling these three authorita. tive volumes and which will be invaluable to investigators dealing with any of the multi-disciplinary aspects of vitaminology. The production of the books is up to the publishers' usual high standards and they will undoubtedly stand up to the continual use they are likely to be subjected to both inside and outside the laboratory.

T. W. Goodwin

\section{NMR PROTON SHIFTS}

NMR Band Handbook

By Herman A. Syzmanski and Robert E. Yelin. Pp. xiv +432. (New York and Washington: IFI/Plenum, 1968.) $\$ 40$.

THrs book is an indexed list of the proton shifts in about 1,200 organic compounds, and, as a typical compound appears several times in the list in respect of each of its distinct proton environments, the list contains 4,800 entries. The material in the volume is a compilation of the data contained in the NMR Spectra Catalog of Varian Associates and Sadtler Research Laboratories, and from NMR Spectral Data of an American Petroleum Institute research project.

A single page of the book consists of twelve entries of the list, each entry displaying the structural formula of the compound with the protons labelled, the shifts referred to the labelled protons, and a coded description of the proton environment to which the entry is specifically related. The proton environment code, which determines the ordering of the entries, is that used in the $N M R$ Spectra Catalog of Varian Associates. It starts with a number designating one of the twenty-one main chemical groups and subsequent upper and lower case letters refer to nearby subgroups and sub-subgroups, respectively. The actual spectral pattern to which the shifts relate is indicated by a type number. The categories are designed to be rather broad, and the possibility of discrepancies between what is predicted and what is actually obtained is admitted. In the light of this, it is unfortunate that no examples of spectra are given in the book. Two indexes are provided: the first is an index of molecular formulae, subheaded into compounds with a list of eppropriate codes; the second is an index of proton shifts in numerical order (p.p.m.), again with the proton codes of the environment which produced them.

The book contains valuable information to aid the determination of structure by providing easy access to data from compounds with features comparable to those of structures which may be proposed. The analyst is also well served for identifying a compound from chemical shifts. A few minor irritations were evident-the usage "comprise" for "compose" in the introduction; $\mathrm{C}_{6} \mathrm{H}_{6}$ appearing in the molecular formula index but not in the body of the book, the fold-out sheet showing proton and peak codes being placed before the indexes rather than after them. The major irritation, however, is the price, which, for a book of this size, containing merely a collection of existent data, makes the prospect of purchase by individuals more than somewhat forbidding. But clearly the handbook will prove a valuable tool for groups working in the field it covers. P. A. Allinson

\section{NUCLEAR MAGNETIC RESONANCE}

Progress in Nuclear Magnetic Resonance Spectroscopy Vol. 2. Edited by J. W. Emsley, J. Feeney and L. H. Sutcliffe. Pp. vii + 269. (Oxford, London and New York: Pergamon Press, Ltd, 1967.) 90s. net.

\section{Advances in Magnetic Resonance}

Vol. 2. By John S. Waugh. Pp. xii +269 . (New York : Academic Press, Inc.; London: Academic Press, Inc. (London), Ltd, 1966.) \$12.00.

\section{Guide to the NMR Empirical Method}

A Workbook. By Roy H. Bible, jun. Pp. $x i+305$. (New York: Plenum Press, 1967.) \$9.50.

IN 1953, the first textbook exclusively devoted to nuclear magnetic resonance (Andrew: Nuclear Magnetic Reson- 